\title{
COMMENT
}

\section{Switching winter and summer photoperiods in an animal model of bipolar disorder}

\author{
Sandra J. Rosenthal (D) and Richard McCarty ${ }^{2}$ \\ Neuropsychopharmacology (2019) 44:1677-1678; https://doi.org/10.1038/s41386-019-0337-4
}

Bipolar disorder $(\mathrm{BD})$ is a severe mental disorder that affects approximately $1-2 \%$ of the population worldwide. The age of onset for this debilitating disorder is approximately 20 years of age, and there is a high rate of suicide attempts associated with $\mathrm{BD}$. Individuals diagnosed with BD may experience significant levels of disability over the lifespan. The heritability of BD is extremely high, with estimates up to $85 \%$, and the genetic architecture is quite complex, with many risk genes thought to be involved, each contributing a small effect. There is also considerable evidence to support a powerful role for environmental factors interacting with these risk genes at different stages of development, including prenatal maternal infections, traumatic experiences in childhood, drug use and abuse, and stressful life experiences. Patients with BD often express co-morbidities for other psychiatric disorders, including attention deficit hyperactivity disorder, anxiety disorders, and substance use disorders [1].

The accidental discovery of lithium for the treatment of BD was first reported by Australian psychiatrist John Cade [2]. Since that time, breakthroughs in the pharmacological treatment of BD have been few and far between. One reason for the relative absence of targeted therapies for BD has been the difficulty in developing valid animal models. Two features of $B D$ that are especially relevant for those interested in modeling this disorder in laboratory animals include the process of switching from a manic or hypomanic episode to depression (or vice versa), and the possible disruption of circadian systems in bipolar individuals, including alterations in sleep-wake patterns and seasonal variations in susceptibility to the onset of mania or bipolar depression. What is clear at this time is that the underlying neural and molecular mechanisms that regulate the switch process are incompletely understood in BD patients, and there is an urgent need for animal models that permit a detailed exploration of these underlying mechanisms.

With this urgent need for improved animal models in mind, Young and colleagues [3] examined seasonal extremes of lightdark cycles on behavioral and neural measures of C57BL/6J mice, WT mice, and mice heterozygous for the dopamine transporter gene (DAT-HZ) that expressed $\sim 50 \%$ of WT levels of DAT protein. Prior to training and behavioral testing, all mice were maintained for at least 2 weeks on 1 of the following 3 photoperiods: longactive (LA, 5:19 light-dark cycle), short-active (SA, 19:5 light-dark cycle), or control (NA, 12:12 light-dark cycle). The time of lights-on for the 3 chambers was adjusted such that they shared a 5-h period of darkness each day when behavioral testing occurred.

DAT-HZ mice housed in a SA photoperiod exhibited increased immobility in the forced swim test, a depression-relevant behavior. In the elevated plus maze, DAT-HZ mice housed in an LA photoperiod displayed more frequent open arm entries, a mania-relevant behavior. In addition, DAT-HZ mice in LA conditions were more sensitive to rewards, while in SA conditions they were more sensitive to punishment and loss in two learning tasks.

Photoperiod also affected the balance between tyrosine hydroxylase (TH)-positive and somatostatin-positive neurons in the hypothalamic paraventricular nucleus (PVN). Compared to mice in the NA photoperiod, DAT-HZ mice had reductions in $\mathrm{TH}$ positive cells but increases in somatostatin-positive cells when housed in an SA photoperiod. The opposite was true for the LA photoperiod, with increases in TH-positive cells and decreases in somatostatin-positive cells. DAT mRNA levels were unaffected by photoperiod in DAT-HZ mice.

We have three major concerns relating to this series of experiments that call into question the conclusions that were drawn. C57BL/6J mice lack the ability to synthesize melatonin, due to a naturally occurring point mutation in the gene coding for serotonin $\mathrm{N}$-acetyltransferase [4]. Pineal melatonin plays a key role in the adjustment of animals to changing photoperiods, and the inability of C57BL/6J mice to synthesize melatonin could possibly affect circadian adjustments in mice of this strain to the extremes of daily light:dark cycles as described in the experiments of Young et al. [3]. In addition, mice were only allowed two weeks to acclimate to the altered photoperiods. It should be noted that VanderLeest and co-workers [5] reported that mice of this same strain required at least 30 days to entrain behaviorally and neurophysiologically to a new long-day or short-day cycle. Finally, these investigators chose to switch the designations for typical winter and summer photoperiods, such that for them, a short-day cycle ( $5 \mathrm{~h}$ light: $19 \mathrm{~h}$ dark) was labeled as "summer-like" (longactive photoperiod) while a long-day cycle ( $19 \mathrm{~h}$ light: $5 \mathrm{~h}$ dark) was labeled as "winter-like" (short-active photoperiod). Increasing the number of hours of darkness in a 24-h period does not necessarily translate into increased daily activity in a nocturnal species such as laboratory mice [6]. Thus, these winter-like and summer-like designations break with well-established conventions of circadian biology and call for a significant re-interpretation of the results presented by Young and colleagues.

The use of DAT knockdown mice and CLOCK mutant mice represents an exciting step forward in the quest for improved animal models of BD. We applaud the creative approach taken by Young and colleagues [3]; unfortunately, the devil is always in the details. We believe some essential features of their experimental design and the interpretation of their results call into question the conclusions that were reached.

\footnotetext{
${ }^{1}$ Departments of Chemistry, Vanderbilt University, Nashville, TN 37235, USA and ${ }^{2}$ Departments of Psychology, Vanderbilt University, Nashville, TN 37240, USA
} Correspondence: Sandra J. Rosenthal (sandra.j.rosenthal@vanderbilt.edu)

Received: 15 December 2018 Revised: 8 January 2019 Accepted: 1 February 2019

Published online: 20 February 2019 
Switching winter and summer photoperiods in an animal model of bipolar... S J Rosenthal and R McCarty

1678

\section{ADDITIONAL INFORMATION}

Competing interests: The authors declare no competing interests.

Publisher's note: Springer Nature remains neutral with regard to jurisdictional claims in published maps and institutional affiliations.

\section{REFERENCES}

1. Vieta E, Berk M, Schulze TG, Carvalho AF, Suppes T, Calabrese JR, et al. Bipolar disorders. Nat Rev Dis Prim. 2018;4:18008.

2. Cade JFJ. Lithium salts in the treatment of psychotic excitement. Med J Aust. 1949;36:349-52.
3. Young JW, Cope ZA, Romoli B, Schrurs E, Joosen A, van Enkhuizen J, et al. Mice with reduced DAT levels recreate seasonal-induced switching between states in bipolar disorder. Neuropsychopharmacology 2018. 2018;43:1721-31.

4. Roseboom PH, Namboodiri MAA, Zimonjic DB, Popescu NC, Rodriguez IR, Gastel $\mathrm{JA}$, et al. Natural melatonin 'knockdown' in C57BL/6J mice: rare mechanism truncates serotonin $\mathrm{N}$-acetyltransferase. Mol Br Res. 1998;63:189-97.

5. VanderLeest HT, Houben T, Michael S, Deboer T, Albus $H$, Vansteensel MJ, et al. Seasonal encoding by the circadian pacemaker of the SCN. Curr Biol. 2007;17:468-73.

6. Schwartz WJ, Zimmerman P. Circadian timekeeping in BALB/C and C57BL/6 inbred mouse strains. J Neurosci. 1990;10:3685-94. 